\title{
Die Begründung der Zellularpathologie durch Rudolf Virchow und die Augenheilkunde
}

\author{
Von M.H.KoelBing ${ }^{1}$
}

Zu Beginn des Jahres 1858 veranstaltete Rudolf ViRchow, damals 36 Jahre alt, für die Ärzte der Stadt Berlin einen Fortbildungskurs in allgemeiner Pathologie, wie wir heute sagen würden. Seine Theorie, die er hier in zwanzig Vorlesungen, verbunden mit mikroskopischen Demonstrationen, entwickelte, war mit dem Namen «Cellular-Pathologie » ein wenig zu knapp umschrieben, ging es ihm doch darum,

«eine Anschauung von der cellularen Natur aller Lebenserscheinungen, der physiologischen und pathologischen, der tierischen und pflanzlichen zu liefern».

Dies freilich in der durchaus praktisch orientierten Absicht,

«gegenüber den einseitigen humoralen und neuristischen (solidaren) Neigungen, welche sich aus den Mythen des Altertums bis in unsere Zeit fortgepflanzt haben, die Einheit des Lebens in allem Organischen wieder dem Bewußtsein näherzubringen, und zugleich den ebenso einseitigen Deutungen einer grob-mechanischen und chemischen Richtung die feinere Mechanik und Chemie der Zelle entgegenzuhalten . $^{2}$

Die Vorlesungen erschienen gegen Ende des Jahres 1858 im Druck und erregten großes Aufsehen in der ganzen wissenschaftlichen Welt, weit über das deutsche Sprachgebiet hinaus. Mit Recht: Gegründet auf einen immensen Schatz sorgfältiger, zum großen Teil eigener Beobachtungen wurde hier eine naturwissenschaftliche Theorie im besten Sinne des Wortes gegeben, das Koordinatensystem gleichsam, in welches sich nun die ungezählten Fakten sinnvoll einordnen ließen, welche das mikroskopische Studium der Gewebe enthüllt hatte und weiterhin noch enthüllen sollte.

Gleich zu Beginn stellt Virchow fest,

1 Dem Virchow-Biographen Herrn Prof. Dr. E. H. Acкerknecht in Zürich bin ich für manche Anregungen und Hinweise zu Dank verpflichtet. Den Herren Prof. Dr. F. RiNtelen, Vorsteher der Universitäts-Augenklinik Basel, und Prof. Dr. A. Werthemann, Direktor der Pathologisch-Anatomischen Anstalt der Universität Basel, danke ich für die großzügige Benützung ihrer Institutsbibliotheken, die sie mir gestattet haben.

2 R. VIrchow, Die Cellularpathologie in ihrer Begrïndung auf physiologische und pathologische Gewebelehre, Berlin 1858 (1. Auflage), Vorrede. 
«daß die Zelle wirklich das letzte eigentliche Formelement aller lebendigen Erscheinung sei, und daß wir die eigentliche Aktion nicht über die Zelle hinausverlegen dürfen ». ${ }^{3}$

Mit dieser Auffassung macht er nun konsequent und kompromißlos ernst; gerade darin dürfte die Durchschlagskraft seiner Theorie zum guten Teil beruht haben.

Die Interzellularsubstanz wird den Zellen zu- und untergeordnet, sie läßt sich in Zellterritorien unterteilen, deren Lebensäußerungen je von einer Zelle oder Zellgruppe abhängen. Die freie Zellbildung aus amorphem Blastem hatte für SchwANn noch die Norm bedeutet, und die Entstehung von Zellen aus den ungeformten Exsudaten des kranken Körpers war für Rokitansky ein Grundphänomen pathologischen Geschehens gewesen. Der Embryologe und Neurologe RemaK hatte diese Möglichkeit zuerst 1852 ganz entschieden negiert, und VIRcHow trieb sie vollends und für immer aus Medizin und Biologie aus:

"Wo eine Zelle entsteht, da muß eine Zelle vorausgegangen sein (Omnis cellula e cellula).»"

Es dürfte kaum möglich sein, im Bereiche des Lebendigen, dessen Geheimnis sich der Forschung nie ganz enthüllt, eine völlig objektive, wissenschaftliche Theorie zu entwickeln, die nicht irgendwie durch die Persönlichkeit des Forschers beeinflußt, durch seine besondere Art, zu fragen, zu sehen und zu denken, subjektiv geprägt wäre. Auch VIRGHow ist es bei allem betonten Bemühen um Objektivität durchaus nicht restlos gelungen. So, wenn wir lesen:

«Jedes Tier erscheint als eine Summe vitaler Einheiten. ${ }^{5}$

Dies ist nicht Beobachtung, sondern Interpretation, nicht Naturwissenschaft, sondern Philosophie. Eine derartig rein additive Anschauung konnte der Ganzheit des Organismus nicht gerecht werden und gestattete notwendigerweise nur ein teilweises Verständnis patho-physiologischer Vorgänge. So etwa bei der Diskussion der Reizungshyperämie, die als Reaktion auf einen das Gewebe treffenden Reiz eintritt und - vom Gesamtorganismus aus gesehen mit Recht - als aktive Hyperämie bezeichnet wird. Nach VIRGHOw ist diese Bezeichnung falsch, da sich die Gefäße dabei vollständig passiv verhalten ${ }^{6}$. Ebenso wird dem Nervensystem das Privileg abgesprochen, als ein einheitlicher Apparat die Einheit des Organismus zu repräsentieren:

3 Cellularpathologie, S. 3.

4 Cellularpathologie, S. 25. - Zur Entwicklung der Zellen-Theorien im allgemeinen s. Ackerknecht, Rudolf Virchow, Stuttgart 1957, S. $58 \mathrm{ff}$.

5 Cellularpathologie, S. $12 . \quad{ }^{6}$ Cellularpathologie, S. 108. 
«Selbst wenn man auch nur ganz grobe anatomische Vorstellungen über die Nerven hat, so sollte man es sich nicht verhehlen, daß es mit dieser Einheit sehr mißlich bestellt ist, und daß schon das Scalpell den Nervenapparat als ein aus außerordentlich vielen, relativ gleichwertigen Teilen zusammengeordnetes System ohne erkennbaren Mittelpunkt darlegt. Je genauer wir histologisch untersuchen, um so mehr vervielfältigen sich die Elemente ... Eine unendliche Menge zelliger Elemente von mehr oder weniger großer Selbständigkeit treten neben und großenteils unabhängig voneinander in die Erscheinung. $\aleph^{7}$

Diese Einseitigkeiten werden verständlich, wenn man daran denkt, daß Virchows Zellularpathologie nicht aus der sublimen Schau eines dem Alltag entrückten, erhabenen Geistes geboren wurde; sie wurde im Gegenteil in unermüdlicher Auseinandersetzung mit den Zeitgenossen geschaffen und erstritten. Die neuen Jahrgänge seines seit 1847 erscheinenden Archivs für pathologische Anatomie und Physiologie und für klinische Medizin pflegte Vırchow jeweils mit einem kämpferischen Beitrag über grundsätzliche Fragen zu eröffnen. In einem solchen «Leit-Aufsatz» hatte er bereits 1855 die Zellularpathologie, welche Solidar- und Humoralpathologie vereinigen und dadurch überwinden werde, als die «Pathologie der Zukunft» proklamiert und gegen mehrere Widersacher verteidigt, darunter vor allem gegen den Frankfurter Arzt Gustav Adolf Spiess ${ }^{8}$. Spiess war - wie viele Mediziner des 18. und früheren 19. Jahrhunderts - von der beherrschenden Rolle des Nervensystems für alles Geschehen im Organismus überzeugt; so anerkannte er beispielsweise im Sinne der damaligen Neuralpathologie als Ausgangspunkt einer Entzündung einzig und allein die krankhafte Erregung der vasomotorischen Nerven ${ }^{9}$.

VIRCHOW dagegen wollte gegenüber der «Aristokratie und Hierarchie von Blut und Nerv» dem "Tiers-état der vielen kleinen Elemente zu seinem Recht verhelfen ». ${ }^{10}$

Das wesentliche Moment für das Entstehen einer Entzündung war für ihn deshalb weder die allfällige Innervationsstörung noch die meist vorhandene Hyperämie, sondern die von den Zellen unter dem Einfluß eines pathogenen Reizes autonom eingeleitete Nutritionsstörung. Entzündung

7 Cellularpathologie, S. 204f.

8 R. VIrchow, Cellularpathologie, Virchows Archiv 8 (1855) 3-40.

9 G. RATH, Der Kampf zwischen Zellularpathologie und Neuralpathologie im 19. Jhdt., Dtsch. Med. Wschr. 82 (1957) 740-3.

10 Die Einheitsbestrebungen in der wissenschaftlichen Medizin, S. 50/51, Fußnote, 1855, zitiert nach RATH. 
kommt auch in nicht innervierten Teilen - Knorpel - und in gefäßfreien Geweben vor:

«Ich kann hier immer nur wieder auf die Mitte der Hornhaut hinweisen... ${ }^{11}$

Dem Leser fällt immer wieder die politische Note auf, die Virchow in seinen wissenschaftlichen Streitschriften anschlägt. Er kämpft für die Anerkennung der Zellen als gleichberechtigter Glieder eines republikanischen Zellenstaates, wie er auf den Berliner Barrikaden des Jahres 1848 für die Gleichberechtigung der Bürger kämpfte. Die Neuralpathologen vertreten ein aristokratisches, monarchisches Prinzip, das für Vıгсноw nicht mehr zeitgemäß ist. Von der Hierarchie des Blutes war vorhin die Rede - es kommt aber noch besser:

«Die Humoralpathologie bedrohte die Medizin wie gleichzeitig der Sozialismus in seiner doktrinärsten Form die Gesellschaft und den Staat»,

schreibt er im Jahre 1861 beim Rückblick auf die Anfänge seines Archivs ${ }^{12}$. Tatsächlich läßt sich bei Virchow der Wissenschaftler vom Politiker nicht trennen, wir sehen uns vielmehr einer imposanten Einheit und Geschlossenheit der Persönlichkeit gegenüber, die sich in Vівсноws gesamtem Wirken und Schaffen erkennen läßt ${ }^{13}$.

Es würde wenig Sinn für Proportionen und damit wenig historischen Sinn überhaupt verraten, wenn man den offensichtlichen Mangel an ganzheitlicher Betrachtungsweise in VIRGHOws Zellularpathologie als verhängnisvoll für die weitere Entwicklung der Heilkunde beurteilen wollte. Nur die Zellularpathologie, die Erforschung der Lebensvorgänge in den kleinsten Aktionselementen, hat es ermöglicht, die Ganzheit des Organismus in den einzelnen Lebensvorgängen wissenschaftlich zu erfassen und für die praktische Medizin erneut fruchtbar zu machen. Hiefür ein Beispiel: Die Lehre des Zürcher Physiologen W. R. Hess und seiner Schule von der funktionellen Organisation des vegetativen Nervensystems mit seinen verschiedenen, einander über- und untergeordneten Aktionsstufen, seinen Regulations- und Gegenregulationsmechanismen, ist das Ergebnis minutiöser Experimente, in welchen die Funktionen einzelner Gruppen von Nervenzellen erforscht wurden.

11 Virchows Archiv 8 (1855) 32.

12 Virchows Archiv 21 (1861) 2.

13 AcKenknecht hat dies eindrücklich herausgearbeitet. 
Ähnliches gilt für die humoralen Steuerungen in Physiologie und Pathologie: Die körpereigenen Wirkstoffe werden von Zellen gebildet und wirken auf Zellen. Die neuere Erkenntnis, daß im Haushalt des Wassers, der Salze und der Kolloide die Interzellularsubstanz eine große und von den Zellen weitgehend unabhängige Rolle spielt, ist eine ergänzende Korrektur von VIrchоws allzu zellulär orientierter Auffassung, nicht aber eine Widerlegung: Die Zellen bleiben die aktiven Elemente im Organismus; auf ihnen bauen sich seit Virchow Physiologie, Pathologie und Pharmakodynamie auf. Nach wie vor bildet die Zellularpathologie das solide Fundament, auf dem die Forscher und Ärzte stehen, auch wenn sie den Blick in den ultramikroskopischen Raum der Moleküle eindringen lassen, oder wenn sie anders orientiert - sich bemühen, den Organismus in seiner Ganzheit mitsamt seinen rätselvollen psycho-physischen Korrelationen zu erfassen.

Die Bedeutung von Virchows Zellularpathologie für seine Zeit lag darin, daß sie die steigende Flut biologischer und pathologischer Forschungsergebnisse ordnend zusammenfaßte, die einzelnen Fakten zur Synthese vereinigte. Diese Synthese, zur rechten Zeit mit Klarheit und Energie verkündet und verteidigt, wurde rasch als der Rahmen anerkannt, in den jeder die Resultate der eigenen Forschungsarbeit einordnen konnte; Virchow lieferte seinen Zeitgenossen gleichsam die Kategorien, in welchen sie hinfort die Wirklichkeit beobachtend und deutend erfassen sollten. Die Beobachtungen jedes einzelnen verflochten sich mit Virchоws Theorie zu neuen Erkenntnissen, in denen die beiden Ingredienzen nicht mehr zu trennen waren und die ihrerseits zur Erweiterung und Vervollkommnung der Theorie dienten. Es ist infolgedessen schwierig, den Einfluß Virchows und seiner Zellularpathologie auf die Teilgebiete der Medizin genauer zu erfassen. Die Ophthalmologie, die sich in der ersten Hälfte des Jahrhunderts bereits zu einem in seiner Selbständigkeit weitgehend anerkannten Spezialfach ausgebildet hatte, dürfte sich hiefür relativ gut eignen. Leichter als dies anderswo möglich ist, läßt sich das Sehorgan dank seinem besonderen Bau mit naturwissenschaftlichen Methoden in seiner Physiologie und Pathologie erforschen. Dieser Umstand machte das Auge für den Pathologen als Studienobjekt und Experimentierfeld interessant und ermöglichte es andererseits dem Arzte, aus der pathologischen Forschung für die Praxis direkten Nutzen zu ziehen. Die Erfindung des Augenspiegels durch HeLm- 
HoLtz (1850) brachte notwendigerweise die beiden Fachgebiete - Pathologie und Ophthalmologie - einander erst recht nahe. Denn nun mußte eine ophthalmoskopische Diagnostik geschaffen werden, die Augenärzte mußten nicht nur lernen, mit dem neuen Instrument zu sehen, sondern auch das Gesehene zu deuten. Hier ist der Punkt, an dem die von VIRchоw so überzeugend formulierte, mikroskopisch-anatomisch so gut begründete Zellularpathologie für die Ophthalmologie aktuellste Bedeutung gewann und wo umgekehrt die Ophthalmoskopie zum Hilfsmittel pathologischer Forschung wurde, indem sie krankhafte Veränderungen an Blutgefäßen, Nervensubstanz (Opticus und Retina) und bindegewebigen Strukturen (Aderhaut) vergrößert in vivo erkennen und in ihrem Verlaufe verfolgen ließ.

Die fruchtbare gegenseitige Durchdringung der beiden Disziplinen wurde schließlich durch die historische Konstellation gefördert. Das Berlin der 1850 er und 1860 er Jahre war nicht nur - durch VIRchow - zur Pflanzstätte der modernen Pathologie geworden, es war zugleich - dank Albrecht v. Graefe - das wichtigste Zentrum der Ophthalmologie. Die «Reformation der Augenheilkunde» (Hirschberg), die zu jener Zeit stattfand, ist gewiß nicht das Werk eines einzigen, aber es ist schon so, daß von v. GraEfe die mächtigsten und vielseitigsten Impulse ausgingen, und daß er in seinem kurzen Leben - als er 1870 starb, war er 42 Jahre alt - den größten persönlichen Beitrag zur Neugestaltung der Augenheilkunde - in Pathologie, Klinik und Chirurgie - geleistet hat. Er war von der Wichtigkeit der pathologischen Anatomie für die ophthalmologisch-klinische Forschung überzeugt und sah zugleich die Einzeldisziplinen im umfassenden Rahmen der Gesamtmedizin. Im Vorwort zur ersten Lieferung seines Archivs für Ophthalmologie (1854) nennt er die Gründe, welche dieses literarische Unternehmen rechtferigen - es sind gleichzeitig die Gründe, welche die Pflege der Augenheilkunde als Spezialfach notwendig gemacht haben:

«Die sich häufenden anatomischen und pathologisch-anatomischen Untersuchungen, die zahlreichen physiologischen Arbeiten, vor allen Dingen aber die Erfindung des Augenspiegels, welche den Namen HeLmholtz mit der Unsterblichkeit schmückt, geben dem Fach einen literarischen Aufschwung, dem selbst der Ophthalmologe nur mit Anstrengung aller Kräfte zu folgen imstande ist. (14 $^{14}$

Die intensive Bearbeitung des Spezialfaches, die Ausnützung der besonderen Möglichkeiten, die dem Ophthalmologen «sein» Organ - das Auge bietet, bereichern zugleich die gesamte Medizin:

14 v. Graefes Archiv für Ophthalmologie 1 (1854) Vorwort. 
«Fassen wir die Hauptvorteile, welche uns die durchsichtige Beschaffenheit des Auges verschafft, zusammen, so liegen sie in der innigen Verschmelzung klinischer und pathologisch-anatomischer Beobachtung, einer Verschmelzung, welche gerade die neuere Medizin mit allen Kräften, aber mit sehr ungleichem Erfolge in den verschiedenen Disziplinen anstrebt. $»^{15}$

Virchow hat v. Graefes Interesse für die Pathologie nach dessen Tod in eindrücklichen, schlichten Worten bestätigt:

«A. von Graefe gehörte im Jahre 1858, als ich diese Vorträge hielt, zu meinen fleißigsten Zuhörern. Ich war ebenso überrascht als gerührt, als ich in diesen Tagen in einem Exemplar der Cellular-Pathologie aus seinem Nachlasse noch die von seiner Hand geschriebenen Notizen fand, in denen er den Gang der Vorträge für sich verzeichnet hatte. ${ }^{16}$

\section{III}

«Für die Entzündungslehre hat das Studium der Cornea die allerwichtigsten Aufschlüsse geliefert», erklärt v.GRAEFE in seiner eben zitierten Rede $^{15}$. VIRchow hatte sich in seiner Inauguraldissertation (1843) mit der «rheumatischen» Keratitis befaßt und dabei einleitend die Bedeutung des Auges für die Erforschung pathologischer Prozesse hervorgehoben:

«In der Tat ist kein Teil des menschlichen Körpers besser geeignet, um Natur und Verlauf der Krankheiten zu studieren, als das Auge, dessen meiste Häute, durchsichtig oder äußerlich gelegen, eine eingehendere Beobachtung gestatten. Auch gibt es keine Membran, keine Verbindung von Geweben, die nicht im Mikrokosmos des Auges vertreten wäre. Doch wenn die Ärzte auch dank diesen Umständen sich alle Mühe gegeben haben, um den Verlauf der Krankheiten zu erkennen, so sehen wir trotzdem, daß die Forschungsmethode, die in jüngerer Zeit von den übrigen Naturwissenschaften auf die Medizin übertragen wurde, beinahe unbeachtet geblieben ist. ${ }^{17}$

Mit der Forschungsmethode, welche die Augenärzte vernachlässigt haben sollen, meint VIRchow - wie aus den weiteren Ausführungen hervorgeht ganz allgemein die naturwissenschaftlich-exakte Analyse der Lebensvorgänge. In erster Linie stehen für ihn dabei noch nicht die mikroskopischanatomischen sondern die chemischen Untersuchungen. Er bemüht sich um eine chemische Theorie des Rheumatismus und nimmt in Übereinstimmung mit der herrschenden humoral-pathologischen Auffassung ein «acre rheu-

15 Rede über die Bedeutung ophthalmologischer Studien für die Medizin, zur Feier des 71. Stiftungstages des med.-chir. Friedrich-Wilhelms-Institutes am 2. August 1865, gehalten von A. v. GRAEFE, Berlin 1865, S. 10/11.

16 Cellular-Pathologie, 4. Auflage, Berlin 1871, S. 379, Fußnote.

17 R. VIRCHow, De rheumate praesertim corneae. Dissertatio inauguralis pathologica... Berlin 1843 , S. 6 . 
maticum " als materia peccans an, das aus dem Blutstrom in den empfänglichen Geweben bei Einwirkung eines lokalen Reizes abgelagert werden soll. Es erübrigt sich, auf diese Theorie, die zum Verständnis des Rheumatismus oder der Keratitis nichts Neues beitragen konnte, weiter einzugehen. Was die Dissertation des 22 jährigen VIRchow vor hundert andern auszeichnet, sind nicht neue Befunde und Erkenntnisse, sondern das umfassende Bestreben, die Errungenschaften der gesamten Naturwissenschaft für die Medizin fruchtbar zu machen - und eine kritiklustige Skepsis gegenüber wissenschaftlichen Autoritäten.

Die von v. GraEfE gefeierten, grundlegenden Erkenntnisse über den Ablauf der Entzündungsvorgänge konnte erst die mikroskopisch-anatomische Untersuchung liefern, und die Cornea hat sich hiefür Virchow und seinen Schülern ${ }^{18}$ als ein Organteil bewährt, der außergewöhnlich günstige Bedingungen für Experiment und Beobachtung bot. Darüber hinaus sah Virchow in ihr - wie schon erwähnt - ein erstklassiges Demonstrationsobjekt für seine These, daß Entzündung nur an das Vorhandensein von Zellen, nicht aber von Blutgefäßen (und Nerven) gebunden sei. Dabei übersieht Virchow keineswegs die Komplexität des Prozesses: Entzündung ist eine Störung der Nutrition der Gewebe; sie hat degenerativen Charakter; sie ist eine Totalerkrankung des betroffenen Teiles, die von einer primären Gefäß- oder Nervenläsion (Hyperämie, Neuralgie), ebensogut aber direkt von einer Gewebsveränderung ausgehen kann ${ }^{19}$.

In der Cornea äußert sich die Entzündung - z. B. nach zirkumskripter Ätzung der Oberfläche - in Anschwellung und Vergrößerung der als Hornhautkörperchen bezeichneten Zellen, es erscheinen Fettmoleküle in den Körperchen, die Kerne vermehren und vergrößern sich. Auch die Interzellularsubstanz trübt sich und erlangt eine mehr fibröse, der Sklera ähnliche Beschaffenheit; in ihr können ebenfalls Fettmoleküle auftreten. Alles in allem findet eine Aufnahme von Material in die Gewebselemente (Hornhautkörperchen und Grundsubstanz) statt, ohne daß irgendwann ein freies Exsudat im Sinne der humoralpathologischen Schule vorhanden wäre; der «Erguß» erfolgt aus den Gefäßen der Conjunctiva direkt in das Gewebe ${ }^{20}$.

18 Fr. Strube, Der normale Bau der Cornea und die pathologischen Abweichungen in demselben. Diss. Würzburg 1851, zitiert in VIRchow, Über parenchymatöse Entzündung, Virchows Archiv 4 (1852) 285. - WILHELM HIs, Beiträge zur normalen und pathologischen Histologie der Cornea, Diss. Basel 1856. - Vor allem aber: F. v. Recklinghausen und J. Cohnherm. - Nachdrücklich verweise ich auf Paul Diepgen, Die Lehre von der Entzündung, von der Begründung der Zellularpathologie bis zum Aufkommen der Bakteriologie, Mainz/Wiesbaden 1953 (Abhandl. der Akad. d. Wissensch. u. d. Lit.)

19 Virchow, Über parenchymatöse Entzündung, Virchows Archiv 4. (1852) 261-324.

20 Virchow, l.c., S. $285 \mathrm{ff}$. 


\section{Virchow verweist auf WiLliam Bowmans frühere Beschreibung ${ }^{21}$ :}

Verletzung der Cornea setzt - nach BowmaN - zunächst eine Ernährungsstörung der betroffenen Partie. Die rasch eintretende Hyperämie der - relativ recht fern gelegenen - Conjunctival- und Skleralgefäße, die dadurch bedingte, vermehrte Zufuhr der für die Heilung nötigen Substanzen zwingen Bowman zur Annahme, daß auch in dem zwischen Gefäßen und Verletzung liegenden Hornhautgewebe und am Orte der Verletzung selbst die nutritiven Vorgänge kräftiger und rascher ablaufen als zuvor. Wenige Stunden nach der Verletzung hat BowmaN bei niederen Tieren die Zellvermehrung in der Umgebung der Wunde und in ihr selbst gesehen:

"The vicinity of the injured part begins to contain in abundance those minute particles, nuclei, or cytoblasts, as they are called, which exist naturally, though sparingly, in the corneal lamellae, and the relative quantity of which may be regarded in most tissues as an index of the intensity of the nutritive function."

Die Körperchen füllen die Gewebslücken in den Wundlippen, überdecken die Wundfläche und bewirken einen vorläufigen Verschluß der Wunde. Diese Vorläufer neuen Gewebes "embryo materials of new tissues" - liegen der zarten, milchigen Trübung zugrunde, die der Arzt an der Verletzungsstelle und in den benachbarten Hornhautpartien wahrnimmt.

Ernährungsstörung des verletzten Gewebsbezirkes, auch wenn dort keine Blutgefäße vorhanden sind, und Zellvermehrung als Zeichen gesteigerter Lebensprozesse und als Vorspiel zur Heilung - diese beiden elementaren Phänomene des Entzündungsprozesses kommen in Bowmans Darstellung sehr schön zum Ausdruck. Ein Beispiel dafür, daß die Zeit für die Zellularpathologie reif war!

Virchows Entzündungslehre wurde vor allem von seinen Schülern Von Recklinghausen (1833-1910) und Cohnheim (1839-1884) weiterentwickelt. Beide haben ausgiebig mit tierischer Cornea experimentiert. VoN Regklinghausen unterschied neben den fixen Bindegewebszellen amöboid sich bewegende «Wanderzellen ». ${ }^{22}$ Besonders schön aber - in Anlage, Durchführung und Darstellung - sind Coнnheims Experimente, mit welchen er die Emigration der Leukozyten aus der Blutbahn nach dem Herd einer Entzündung nachwies ${ }^{23}$. Er injizierte feindisperses Anilinblau in die Lymphsäcke des Frosches, dann auch direkt in die Aorten; der Farbstoff wird von den Leukozyten aufgenommen; die derart «gefütterten» Zellen konnte Сонnнeiм am Orte der experimentell gesetzten Entzündung im

21 William Bowman, Lectures on the Parts Concerned in the Operations on the Eye and on the Structure of the Retina, delivered... 1847, London 1849, S. 28-30.

22 Diepgen, l.c., S. 8.

23 J. Cohnнeim, Über Entzündung und Eiterung, Virchows Archiv 40 (1867) 1-79. Die Diapedese der Leukozyten durch die Gefäßwand war schon von verschiedenen Forschern vorher gesehen worden, s. AcKerknecht, l.c., S. 54, Fußnote. 
Zentrum der Cornea wiederfinden. Dies stand freilich im Gegensatz zu Virchows ursprünglicher, auch von His übernommener Ansicht, daß die ortsansässigen Zellen des vom Entzündungsreiz getroffenen Bindegewebes allein an der entzündlichen Zellvermehrung beteiligt seien. Gleichzeitig gewann durch Coнnherms Beobachtungen der Blutkreislauf, die Gefäße, erneut eine größere Bedeutung im Entzündungsprozeß: «ohne Gefäße keine Entzündung! \" ${ }^{24}$ VIRchows frühere Widersacher waren damit - wenigstens in diesem Punkte - durch VIRchows Schüler rehabilitiert worden. Aber welche Verfeinerung der Kenntnisse, welche Vertiefung des Verständnisses - dank VIRchows Zellularpathologie!

\section{IV}

Während die mit Epithel bekleidete Bindegewebsplatte der Cornea ihre Verwandtschaft mit anderen bindegewebigen Formationen leicht erkennen läßt, zeichnen sich zwei weitere Strukturen, die ebenfalls zu den brechenden Medien des Auges gehören, durch ihre Einzigartigkeit aus: die Linse und der Glaskörper.

Die Entwicklung der Linsenfasern aus ektodermalen Zellen wurde in den $1830 \mathrm{er}$ und $40 \mathrm{er}$ Jahren von verschiedenen Forschern erkannt ${ }^{25}$. Hingegen muß es für VIRchow ein kleiner Triumph gewesen sein, als er auch in der fast flüssigen, scheinbar strukturlosen Gallerte des Glaskörpers Zellen finden konnte ${ }^{26}$. Damit war auch dieses absonderliche Gebilde zu einer Provinz des universalen Zellenstaates geworden. Seine erste Beobachtung der Glaskörperzellen machte Virchow am Auge eines Schweine-Embryos und beschrieb diesen embryonalen Glaskörper als typisches Schleimgewebe, wie es sich auch in Chorion und Nabelstrang vorfinde. An der bindegewebigen, mesenchymalen Natur des Corpus vitreum gab es für Virchow natürlich keinen Zweifel. In der Folge hat sich aber das Problem als wesentlich heikler erwiesen, und die modernste Theorie läßt den Glaskörper aus den Basalmembranen der embryonalen Linsen- und Netzhaut-Epithelien entstehen ${ }^{27}$.

24 J. Cohnheim, l.c., zitiert nach Gesammelte Abhandlungen, Berlin 1885, S. 241.

25 Ch. Dejean nennt Valentin 1835, Schwann 1839, Vogt 1842, ferner Remak und Nussbaum: Ch. Dejean, Fr. Hervouet, G. Leplat, L'embryologie de l'ceil et sa tératologie, Paris 1958, S. 309.

26 R. Virchow, Notiz über den Glaskörper, Virchows Archiv 4 (1852) 468. - Über den menschlichen Glaskörper, ibid. 5 (1853) 278. - R. A. KöLlıker hat um die gleiche Zeit Virchows Befund bestätigt.

27 Ch. Dejean, l.c., S. 220-308. 
Wenden wir uns nun der Frage zu, welchen Gewinn die praktische Ophthalmologie aus Virchows Pathologie zog. Es ist klar, daß die zellularpathologische Betrachtungsweise für das Verständnis der Refraktionsanomalien, die als Formfehler des Auges in seiner Gesamtheit aufzufassen sind, keine neuen Momente zu bieten hatte. Gleiches gilt für die Koordinationsstörungen des binokularen Sehens, das Schielen. Auch die Abnormitäten des Linsenstoffwechsels, die zum grauen Star führen, entziehen sich naturgemäß der mikroskopisch-anatomischen Erforschung. Für die Genese einer besonderen Gruppe von Starformen enthielt die Zellularpathologie freilich einen Schlüssel zum Verständnis: nämlich für die Cataracta congenita und die während der Entwicklungsperiode auftretenden Linsentrübungen. Form und Ausmaß der Katarakte hängen in diesen Fällen davon ab, in welchem Zeitpunkt der embryonalen (oder postnatalen) Entwicklung die faserbildenden Epithelzellen der Linse geschädigt werden.

Das Glaukom beruht nach unserem heutigen Wissen auf einer gestörten Regulation der Kammerwasserzirkulation, die zur Steigerung des intraokularen Druckes führt. Dabei kann die Sekretion von Kammerwasser durch das Corpus ciliare vermehrt oder - was meist der Fall ist - der Abfluß aus der Vorderkammer in die episkleralen Venen erschwert sein. Trotz imposanten Detailkenntnissen, zu deren Mehrung in jüngster Zeit die Berner Schule von Goldmans sehr viel beigetragen hat, sind wir bis jetzt noch weit entfernt von einer umfassenden und befriedigenden pathogenetischen Theorie des primären Glaukoms.

Die Betrachtungsweise der reinen Zellularpathologie mit ihrer Überbetonung der lokalen Vorgänge konnte einem so komplexen Leiden nicht gerecht werden. In etwas merkwürdiger Anwendung von Virchows Auffassung, daß alle Ernährungsvorgänge einschließlich des Flüssigkeitsaustausches einzig durch die Zellen reguliert würden, machte KLEBS die Zellen des Glaskörpers, oder genauer gesagt deren Atrophie, für die «Anhäufung von Flüssigkeit» und damit für eine Drucksteigerung im Glaskörperraum verantwortlich $^{28}$. Er stützte diese These wohlverstanden auf Experimente, die ihm gezeigt hatten, daß der Druck im Glaskörperraum höher sein könne als in der Vorderkammer des Auges - ein Beispiel für die heute allzuwenig berücksichtigte Tatsache, daß Experimentieren nicht vor Spekulieren schützt!

${ }^{28}$ KLeBs, Zur normalen und pathologischen Anatomie des Auges (1.Teil), Virchows Archiv 19 (1860) 349-50. 
Auch v. Graefe sah die Ursache der Drucksteigerung in der übermäßigen Produktion von intraokularer Flüssigkeit; Theodor LeBer (1840-1917), einer seiner bedeutendsten Schüler, rückte dann jedoch die Rolle des Kammerwasser-Abflusses und seiner Behinderung in den Vordergrund.

Das Studium der Entzündungsprozesse am Auge beschränkte sich nicht auf die Cornea. C. O. Weber, Chirurg und Pathologe in Bonn, erzeugte experimentell entzündliche und degenerative Veränderungen im Glaskörper; bei diesen "pathologischen Ernährungsstörungen» konstatierte er «die vollkommenste Analogie mit denen anderer gefäßloser Gewebe». ${ }^{29}$ Ein für die Praxis besonders wichtiges Ergebnis dieser Versuche lag im Nachweis, daß das uralte Verfahren des Starstiches, die Reklination der Linse nach hinten-unten, also in den Glaskörperraum, trotz der kleineren Operationswunde viel leichter als die Extraktion zu Trübung und Vereiterung des Glaskörpers führe: «Selbst eine schlecht ausgeführte Extraction ist für den Glaskörper weniger gefährlich als eine Reclination. » ${ }^{30}$

Seit der Einführung der Star-Extraktion durch Daviel waren zwar schon über hundert Jahre vergangen; trotzdem hatte sich das neuere und bessere Verfahren noch nicht ganz durchgesetzt: Auf JüngKens Abteilung an der Berliner Charité wurde noch in den 1860 er Jahren rekliniert.

Die pathologischen Erscheinungen am Augenhintergrund stellten Ophthalmologen, Pathologen und Internisten zugleich vor faszinierende Probleme, denken wir nur an die Stauungspapille, Embolie und Thrombose der Netzhautgefäße, die Netzhautveränderungen bei Nierenleiden ${ }^{31}$. Das Archiv für Ophthalmologie brachte zu v. Graefes Lebzeiten fast Jahr für Jahr wirkliche Entdeckungen auf dem klinisch-ophthalmologischen und histo-pathologischen Gebiet. Dazu gesellten sich chirurgische, optische und sinnesphysiologische Beiträge, so daß diese Bände tatsächlich Medizingeschichte in ganz konzentrierter Form enthalten ${ }^{32}$. Albrecht v. Graefe,

${ }^{29}$ C. O. Weber, Utber den Bau des Glaskörpers und die pathologischen, namentlich entzündlichen Veränderungen desselben, Virchows Archiv 19 (1860) 367-431.

30 l.c., S. 426.

31 Als Internist arbeitete Ludwig Traube (1818-1876) mit Virchow und v. Graefe zusammen.

32 In diesem Zusammenhang muß Heinrich Müller (1820-1864) erwähnt werden, der seit 1847 in Würzburg lehrte, mit KöLliKer, Virchow und v. GraEFE zusammenarbeitete und sich um die mikroskopische Anatomie des gesunden und kranken Auges sehr verdient gemacht hat; cf. J. Hrrschberg, Geschichte der Augenheilkunde (GraefeSaEmisch, Handbuch der gesamten Augenheilkunde, 2. Auflage, Vol.12/15, 1899-1918), Paragraphen 1181-1183. 
bei der Erforschung des Fundus oculi der führende Geist, verzeichnete jeweils aufs genaueste die Verdienste aller seiner Mitarbeiter und früherer Untersucher, frei von aller Prioritätssucht und jeder Sorge um den eigenen Ruhm. Ein typisches Beispiel für dieses «team work» besitzen wir u.a. im Nachweis der miliaren Tuberkel der Aderhaut als eines pathognomonischen Zeichens der Miliartuberkulose; es war das einzige, bevor die RönTgeNStrahlen die miliare Aussaat in den Lungen sichtbar machten: von GraEFE, LEBER und CoHNheIm haben gemeinsam durch minutiöse Untersuchungen die Identität der ophthalmoskopisch festgestellten Aderhaut-Herdchen mit autoptisch und mikroskopisch verifizierten Miliartuberkeln bewiesen ${ }^{33}$.

Die schönste Frucht der Zellularpathologie in unserem Fachgebiet war jedoch die neue, histogenetisch begründete Lehre von den Geschwülsten, besonders von den so gefährlichen, intraokularen Tumoren ${ }^{34}$. Bei diesen handelt es sich fast ausnahmslos um das heimtückische Melanom der Uvea oder um das äußerst bösartige Retinoblastom, das in frühester Kindheit auftritt. Von Virchоw wurden diese Geschwülste - wie alle Tumoren, wenn immer es ging - dem Bindegewebe zugeordnet; unser heutiges Retinoblastom ist sein Glioma retinae. Zu Beginn des 19. Jahhrhunderts kannte man makroskopisch-anatomisch und klinisch diese Neubildungen in ihrer Bösartigkeit durchaus, ob man nun - mit LAËNNEG - von "mélanose» und "encéphaloïde » sprach oder mit WARDROP von Fungus haematodes oder auf gut deutsch von weichem Krebs, Blut- oder Markschwamm. Die nun folgende Periode mikroskopischer Untersuchungen führte zunächst keineswegs zur besseren Kenntnis ihrer Natur. Denn man war gewohnt, eine spezifische Dyskrasie als Ursache des Krebses anzunehmen und glaubte deshalb, unter dem Mikroskop auch spezifische Krebszellen finden zu müssen. Da man aber keine fand, tauchten Zweifel an der krebsartigen Natur und damit an der obligaten Malignität dieser Tumoren auf. VIRchow brachte hier die nötige Ordnung und Klärung, indem er die Abstammung sämtlicher Neubildungen von normalen Körperzellen zum Gesetz erhob.

Die Geschwulstlehre ist damit zugleich derjenige Bereich der Ophthalmologie, in welchem die Zellularpathologie für die Therapie unmittelbaren Nutzen brachte: Die vitale Notwendigkeit der operativen Entfernung eines

33 A. v. Graefe und Th. Leber, Über Aderhauttuberkeln, v. Graefes Archiv f. Ophth. 14/I (1868) 183-206. - Die erste Beschreibung von Aderhaut-Tuberkeln hat W. MaNz in Freiburg im Breisgau 1858 veröffentlicht: v. Graefes Archiv 4/II (1858) 120-6.

34 Cf. M. H. Koelbing, Die Pathologie intraokularer Tumoren von Fabricius Hildanus bis Virchow und Graefe, Klin. Mbl.f. Augenheilk. 126, (1955) 82-8. 
tumor-befallenen Auges war nun außerhalb jeden Zweifels festgestellt. Ganz allgemein mußte die Zellularpathologie mit ihrem fast ausschließlichen Interesse für die lokalisierten Prozesse logischerweise die lokalen, therapeutischen Maßnahmen besonders sinnvoll erscheinen lassen, auf die Allgemeinbehandlung jedoch einen hemmenden Einfluß haben: Da der Sitz der Erkrankungen aus den Körpersäften in die Zellen verlegt worden war, hatten die bisher geübten, humoraltherapeutischen Methoden ihre Berechtigung zum guten Teil verloren. Dies hatte nun freilich auch sein Gutes: Die intensive «ableitende» Behandlung mit erschöpfenden Purgationen, ausgiebigstem Blutentzug durch Aderlässe und Blutegel, den scheußlichen Haarseilen und Cauterien bedeutete doch oft eine nutzlose und nicht selten eine schädliche Plagerei des Patienten. Die heilsame Skepsis gegenüber solchen Methoden artete jedoch bei VIRchow nicht in jenen «therapeutischen Nihilismus » aus, der merkwürdigerweise gerade die von RokItANSKY inspirierte Wiener Klinik unter SKоDA kennzeichnete. Selbst als ordentlicher Professor der Pathologie in Berlin war VIRсноw noch als Arzt tätig und behandelte mit Erfolg gelegentlich auch kranke Augen, wie wir aus seinem Aufsatz «Über die Natur der constitutionell-syphilitischen Affectionen » ${ }^{35}$ wissen. Dort ist u.a. von der syphilitischen Iritis die Rede; Gegner der antiluetischen Quecksilberbehandlung vertraten damals noch die Ansicht, die Iritis und andere Manifestationen der sekundären und tertiären Syphilis seien weniger durch die Krankheit selbst als vielmehr durch das Quecksilber verursacht. Gegen diese These führt VInchow die Heilerfolge der Quecksilbertherapie ins Feld:

«Selbst die Form mit markiger (gummöser?) Knotenbildung habe ich durch eine streng mercurielle Behandlung in 14 Tagen vollständig beseitigt. $»^{36}$

Er möchte dem alten Streit zwischen Mercurialisten und Antimercurialisten ein Ende setzten: Es sei damit dieselbe traurige Geschichte wie beim Aderlaß - wozu die Pendelbewegung noch einmal wiederholen?

«Handelt es sich nicht vielmehr darum, die Grenzen des Mercurgebrauches zu finden, die Garantien gegen seine unleugbaren Gefahren aufzusuchen ? \ $^{37}$

Die Arbeit ist nicht nur ein eindrückliches Plädoyer für die zielbewußte, kontrollierte Anwendung des Quecksilbers zur Behandlung der Lues; sie

35 Virchows Archiv 15 (1858) 217-336.

36 l.c., S. 307.

37 l.c., S. 331. 
dokumentiert darüber hinaus in überzeugender Weise, daß für Vircноw die Pathologie nicht Selbstzweck war, sondern Dienerin der ärztlichen Praxis sein sollte.

Der Versuch, dem Einfluß von Virchows Zellularpathologie auf die Ophthalmologie nachzugehen, hat uns Pathologie und Augenheilkunde in einer Epoche schönsten Aufblühens gezeigt und ließ uns erkennen, wie die beiden Wissensgebiete einander gegenseitig befruchtet und bereichert haben. Eine unter den Ursachen dieser glücklichen Wechselwirkungen erscheint besonders erfreulich, und sie ist gewiß nicht die unwichtigste: das gute Einvernehmen und die neidlose Zusammenarbeit der beteiligten Forscher unter der Führung von Rudolf Virchow und Albrecht von Graefe. 